\title{
0142 SANITARY CONDITION OF LIFE AND CHILDREN'S HEALTH
}

G Otgon, A Ganbold* Correspondence: National Dermatological Center, Ulaanbaatar, 210648, Mongolia

\subsection{6/ip.2010.029215.142}

To develop of bronchitis and pneumonia of young aged childrens and diseases of cardiovascular system (CVS) and urinogenital system - of later aged childrens influenced sanitary conditions of life. In Mongolia had 541149 family, lives in ger and houses $-50.9 \%$ and in apartment $-49.1 \%$.

Aim The aim of research is to reveal of possible risk factors, such as sanitary household conditions of life on disease of respiratory system (RS), CVS and urinogenital system among children of Mongolia.

Methods Used accounting data of health of Ministry of Health of Mongolia and the Centre of Governmental Statistics. Statistical processing is made on regression analysis on the program SPSS 11.

Results of research High risk for developing bronchitis and a pneumonia of children was residence in ger, absence of the centralised heating $(p \leq 0.001)$ and impossibility of maintenance of optimum parameters of the microclimate $(p \leq 0.005)$ and electrification $(p \leq 0.02)$, kind of using fuel $(p \leq 0.005)$, absence of the centralised removal of liquid waste $(p \leq 0.004)$, absence of the electric power $(p \leq 0.01)$ render system of removal of a withdrawal of residing, as removal on a garbage tank $(p \leq 0.01)$. Increase of disease of urinogenital system of children on later age is connected with local heating $(p \leq 0.0009)$ and transporting water supply system $(\mathrm{p} \leq 0.02)$.

Conclusion Hygienic conditions of residing of the population of Mongolia cause risk of development diseases of RS, such as a pneumonia, a bronchitis. The most significant risk factors are: absence of central heating, the electric power, kind of used fuel for local heating. 